
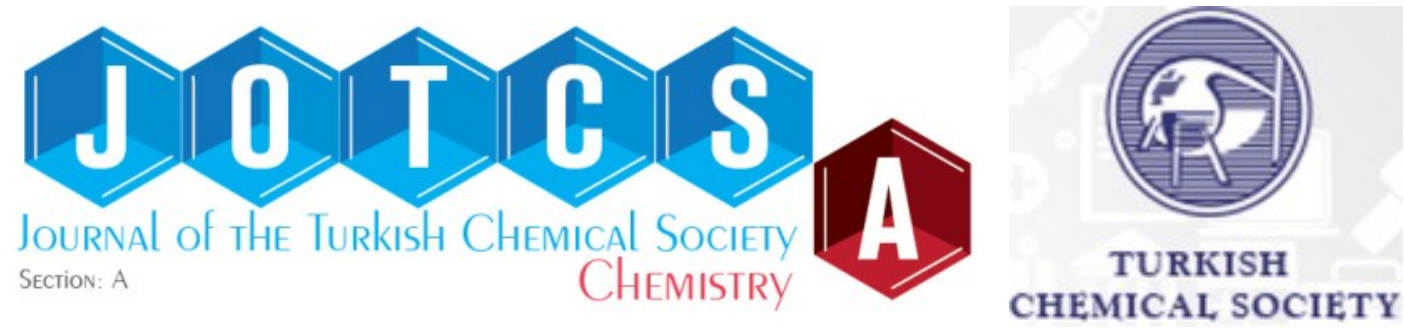

\title{
In situ Crosslinking System of Gelatin with Acrylated $\beta$-cyclodextrin Towards the Fabrication of Hydrogels for Sustained Drug Release
}

\author{
Mehmet ARSLAN ${ }^{1} *$ \\ ${ }^{1}$ University of Yalova, Faculty of Engineering, Department of Polymer Materials Engineering, 77200, Yalova, \\ Turkey.
}

\begin{abstract}
Cyclodextrins (CDs) are of interest in the fabrication of various polymeric platforms, especially the crosslinked networks that possess unique advantages in biomedical applications. When one benefits from the abilities of CDs to form inclusion complexes with hydrophobic drug molecules, hydrogel-based supported drug platforms employ CDs as nano-buckets in the network structure. In the current study, we report on the fabrication of gelatin-based hydrogels chemically crosslinked with acrylated $\beta$-cyclodextrin ( $\beta$ CD) as sustained drug release platforms. A practical and straightforward synthesis of crosslinked networks was achieved by Michael's addition reaction of gelatin amino functionalities onto acryloyl groups of modified $\beta-C D$. Tunable synthesis of hydrogels incorporating a different amount of $\beta-C D$ molecules was maintained by simple adjustment of the reaction stoichiometry between amine and acrylate groups. The resulting hydrogels were characterized by their surface morphology, equilibrium swelling ratios, and rheological properties. Thus obtained hydrogels were evaluated in terms of their drug loading capacities and sustained release efficiencies. The results demonstrated that the amount of drug loading and prolonged release is dependent on the amount of $\beta-C D$ in the gel network. Thanks to their in situ gel-forming abilities, these hydrogels can be used as injectable formulations for various biomedical applications.
\end{abstract}

Keywords: Drug releasing hydrogels, $\beta$-cyclodextrin, aza-Michael addition, injectable hydrogels.

Submitted: April 10, 2020. Accepted: June 07, 2020.

\section{Cite this:}

DOI: https://doi.org/10.18596/jotcsa.717856.

*Corresponding author. E-mail: mehmet.arslan@yalova.edu.tr, Tel: +90-226-811-5959.

\section{INTRODUCTION}

Hydrogels purvey some unique and advantageous properties that make them ideal candidates in several biomedical applications. (1-3) Significant research has been devoted to engineered hydrogels in practical clinical utilization, such as in controlled/sustained drug release platforms or tissue construction scaffolds. In tissue engineering, hydrogels are employed as environments for adhesion, encapsulation, growth, or directed spread of cells. (4-6) In therapeutic applications, hydrogels are exploited as slow drug releasing platforms in which the rate of release can be controlled via response toward external stimuli such as temperature or $\mathrm{pH}$ of the local environment. (7-9) While the eximious features of hydrogels are advantageous, the challenges associated with the practical introduction of the hydrogels into the body might limit their routine clinical implementation. Surgical implantation of the pre-formed hydrogels to defect sites is often associated with patient compliance and discomfort. In this context, noninvasive techniques such as employment of injectable hydrogel formulations to administrate regenerative factors or therapeutics to the body have been addressed. (10-12) In situ cross-linkable hydrogels that can form the network structure in vivo through physical or chemical interaction of gel, precursors could allow straightforward inclusion of drugs, (13) growth factors (14) or genes (15) to the target body tissues.

Gelatin is a biopolymer of animal-origin, derived from collagen by partial hydrolysis and denaturation. Due to essential characteristics such 
as excellent biocompatibility, nontoxicity, high resemblance to extracellular matrix in a gel state, and low immunogenicity make gelatin widely used in the biomedical fields. (16-18) Gelatin contains peptide sequences that can bind to cell surface integrins, which is notable for cellular recognition. (19) Improved cell attachment and proliferation make gelatin-based scaffolds ideal for tissue regeneration, cell encapsulation, and 3D cell culture applications. (20-22) The utility of gelatin-based controlled-release systems covers a wide variety of drug and gene delivery applications. (23) Although gelatin can form temperature-induced physical gelation, a much-sought strategy is the fabrication of chemically crosslinked hydrogels to increase the stability of the gels. Toward this, several injectable gel-forming systems such as dextran-gelatin hydrogels via oxidized dextran and gelatin, (24) gelatin polymers with pendant norbornene (GeIN) or tetrazine (GelT) groups,(25) and UV-curable acrylated gelatin hydrogels (26) have been reported.

Cyclodextrins (CDs) are torus-shaped molecules that are derived mainly degradation of starch. The structures of CDs possess an interesting molecular property with a hydrophobic inner cavity and hydrophilic outer structure. CDs can form inclusion complexes through the hydrophobic inner cavities with molecules that are physically compatible with the cavity size. The dynamic inclusion complexation abilities of CDs with low polarity organic compounds make them ideal materials in the fabrication of biomaterials augmenting the properties in various applications. $(27,28)$ To date, CDs have been employed in the fabrication of diverse hydrogelbased polymeric drug delivery systems, especially to modify the release kinetics. (29-32) While the overall hydrophilic nature of $\mathrm{CDs}$ increases the swelling state of the network structure, the hydrophobic inner core enables enhanced loading and prolonged/sustained release of hydrophobic therapeutic compounds.

In this contribution, we report on the synthesis of gelatin-based hydrogels incorporating $\beta$-cyclodextrin residues towards sustained drug release applications. Briefly, hydrogels were prepared via reacting gelatin amino groups with acrylate modified $\beta-C D$ in aqueous buffer solutions (Figure 1 ). The method demonstrated a facile and efficient crosslinking process via aza-Michael addition of complementary functional precursors, which can be employed as an injectable gel-forming system. A series of hydrogels displaying tunable physical properties were obtained by changing the stoichiometric balance between gelatin amino groups and acrylate modified $\beta-C D$ crosslinker. Drug loading and sustained release studies of fabricated hydrogels were investigated by using poorly watersoluble anti-inflammatory drug diclofenac sodium. The results indicated that the amount of drug loading and release profiles could be controlled by changing the $\beta-C D$ crosslinker ratio. It is believed that the benign gelation methodology outlined here can be utilized in the design and synthesis of bulk and injectable hydrogels for tissue engineering, drug delivery, and various other biomaterial applications. 


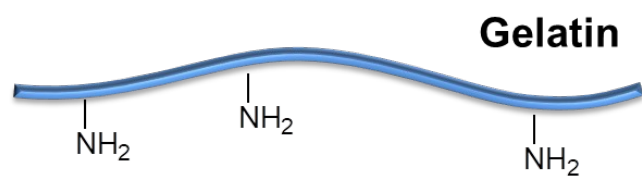

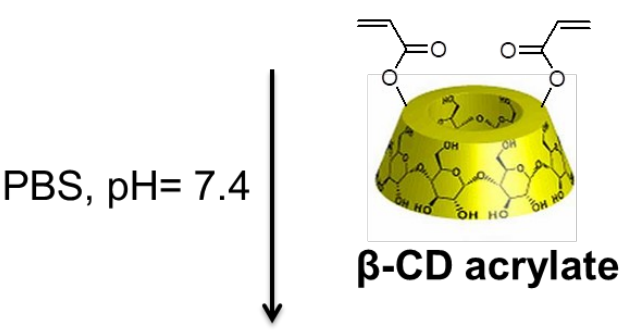
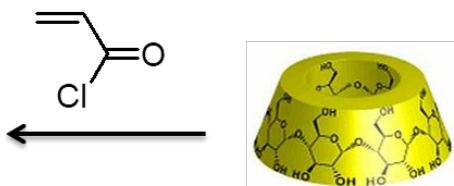

$\beta-C D$

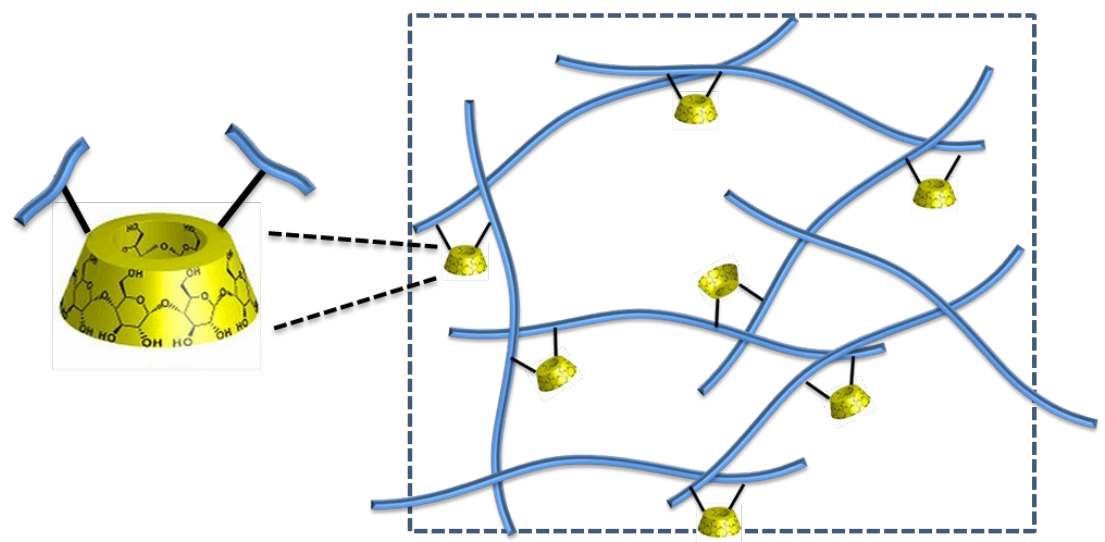<smiles>O=C(Cc1ccccc1Nc1c(Cl)cccc1Cl)O[Na]</smiles>

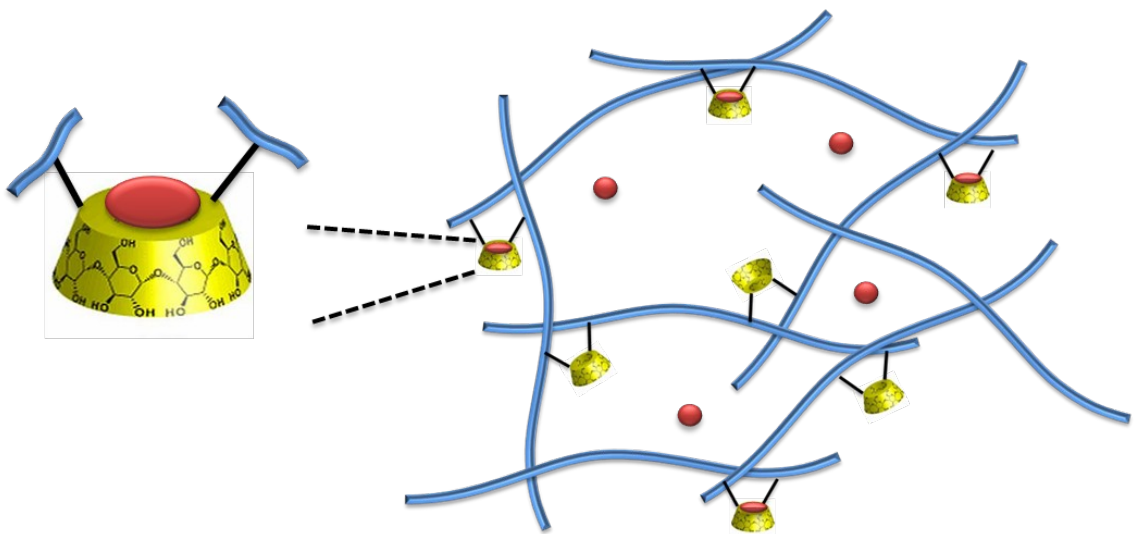

Figure 1. Schematic representation of the gelatin-based hydrogel synthesis with a $\beta$-CD-based crosslinker and the diclofenac Na loading into the hydrogel augmented by the inclusion complex formation.

\section{EXPERIMENTAL SECTION}

\section{Materials and characterization}

$\beta$-cyclodextrin, acryloyl chloride, diclofenac sodium salt, 1,8-diazabicyclo(5.4.0)undec-7-ene (DBU), gelatin from porcine skin (type $A, 300 \mathrm{~g}$ bloom, average $M_{n}: 50.000-100.000 \mathrm{~g} / \mathrm{mol}$ ) were obtained from Aldrich Chemical Co. All solvents and inorganic materials were obtained from Merck Co. and used as received unless otherwise noted. Synthesis of acryloyl-modified $\beta$-cyclodextrin ( $\beta$-CDacrylate, the average degree of acetylation $\sim 5.9$ 
per molecule. Detailed synthesis procedure is given in Supporting Information) was carried out based on the reported procedure. (33) Characterization of $\beta$ $\mathrm{CD}$ acrylate was performed using ${ }^{1} \mathrm{H}$ NMR spectroscopy (Varian $400 \mathrm{MHz}$ ). Fourier-transform infrared spectroscopy analyses were conducted on a Nicolet 380 spectrometer. Scanning electron microscopy analysis was carried out using an ESEMFEG/EDAX Philips XL-30 (Philips, Eindhoven, The Netherlands) instrument with $10 \mathrm{kV}$ accelerating voltage. The rheological measurements were performed using an Anton Paar MCR 302 rheometer between $0.05-100 \mathrm{rad} / \mathrm{s}$ at $37{ }^{\circ} \mathrm{C}$. UV-vis studies were carried out using a Varian Cary 50 Scan UV/Vis spectrophotometer.

\section{Methods}

Synthesis of gelatin-based $\beta-C D$ incorporated hydrogels

In a vial, gelatin (100 mg) was placed and dissolved in $1 \mathrm{~mL}$ PBS buffer (phosphate-buffered saline, $\mathrm{pH}$ of 7.4) at $37{ }^{\circ} \mathrm{C}$. In a separate vial, a predetermined amount of $\beta-C D$-acrylate and DBU ( 0.1 molar equivalents to acrylate groups) were placed and dissolved in $200 \mu \mathrm{L}$ of PBS solution. The twoingredient solutions were thoroughly mixed and sonicated briefly. The mixture solution was heated at $37{ }^{\circ} \mathrm{C}$ for $6 \mathrm{~h}$ for a crosslinking reaction. After that, the resultant hydrogel was washed with distilled water several times to remove unreacted species. Dried hydrogels were obtained after the freeze-drying of water-swollen samples.

\section{Equilibrium swelling ratios (ESRs)}

Equilibrium swelling of hydrogels in distilled water was determined at $25^{\circ} \mathrm{C} .20 \mathrm{mg}$ hydrogel was conditioned in distilled water, and the mass increase of sample as a function of time was recorded until a constant weight was attained. The percent swelling was determined using empirical equation 1 :

$$
\operatorname{ESR}(\%)=\left(W_{\text {wet }}-W_{\text {dry }}\right) / W_{\text {dry }} \times 100
$$$$
\text { (Eq. 1) }
$$

The swelling tests were performed in triplicate for each sample, and average data with standard deviation was used for obtaining swelling curves.

\section{Rheological characterization}

The rheological properties of hydrogels were studied by dynamic oscillatory experiments at a constant 1
$\%$ strain. Loss $\left(G^{\prime \prime}\right)$ and storage $\left(G^{\prime}\right)$ moduli of disk-shaped water-equilibrated hydrogels were measured at $37^{\circ} \mathrm{C}$ between 0.05 and $100 \mathrm{rad} / \mathrm{s}$ angular frequency. $8 \mathrm{~mm}$ diameter parallel plate was used for measurements and the plate distance was set to $2.0 \mathrm{~mm}$.

\section{Drug loading and release studies}

Diclofenac-Na was loaded into the hydrogels by employing solution absorption. Briefly, $50 \mathrm{mg}$ water-swollen gel sample was soaked in 0.5 wt.\% drug solution in PBS ( $\mathrm{pH}: 7.4)$. The hydrogel was incubated at $37{ }^{\circ} \mathrm{C}$ for $24 \mathrm{~h}$, protected from light. Total drug loading was determined from the concentration difference between initial and final soaking solutions using a UV-Vis spectrophotometer (276 nm absorption maxima of diclofenac-Na in PBS solution).

Drug release profiles of hydrogels were investigated by immersing gel samples in $3 \mathrm{~mL}$ of PBS solution at $37{ }^{\circ} \mathrm{C}$. Aliquots of the release medium $(1 \mathrm{~mL})$ were taken out and replaced with the new solution at predetermined intervals. The total drug content in the collected medium was determined spectrophotometrically at $276 \mathrm{~nm}$. The experiments were performed triplicate, and the release profiles were expressed in terms of cumulative release.

\section{RESULTS AND DISCUSSION}

\section{Synthesis and characterization of gelatin- based $\beta-C D$ containing hydrogels}

Hydrogels composed of a gelatin matrix and distinct $\beta-C D$ units were fabricated via amine-acrylate Michael addition of gelatin amino functionalities onto acryloyl groups of modified $\beta-C D$ (Figure 2 ). Though it may vary depending on the source, the amino acid composition of gelatin mostly consists of glycine, proline, and hydroxyproline. Aminecontaining lysine residues also constitute the structure of both bovine and porcine skin gelatin. (34) In chemical derivatization and modification of gelatin, amino groups of lysine have been exploited in reactions with isocyanates, (35) aldehydes, (36) vinyl sulfones(37), and genipin. (38) Towards the synthesis of gelatin-based hydrogels, reactive amino groups of amino acid chain strands were utilized in crosslinking reaction with activated carbonyls (39) as well as a modification with photo-curable acrylate functionalities. (26) 

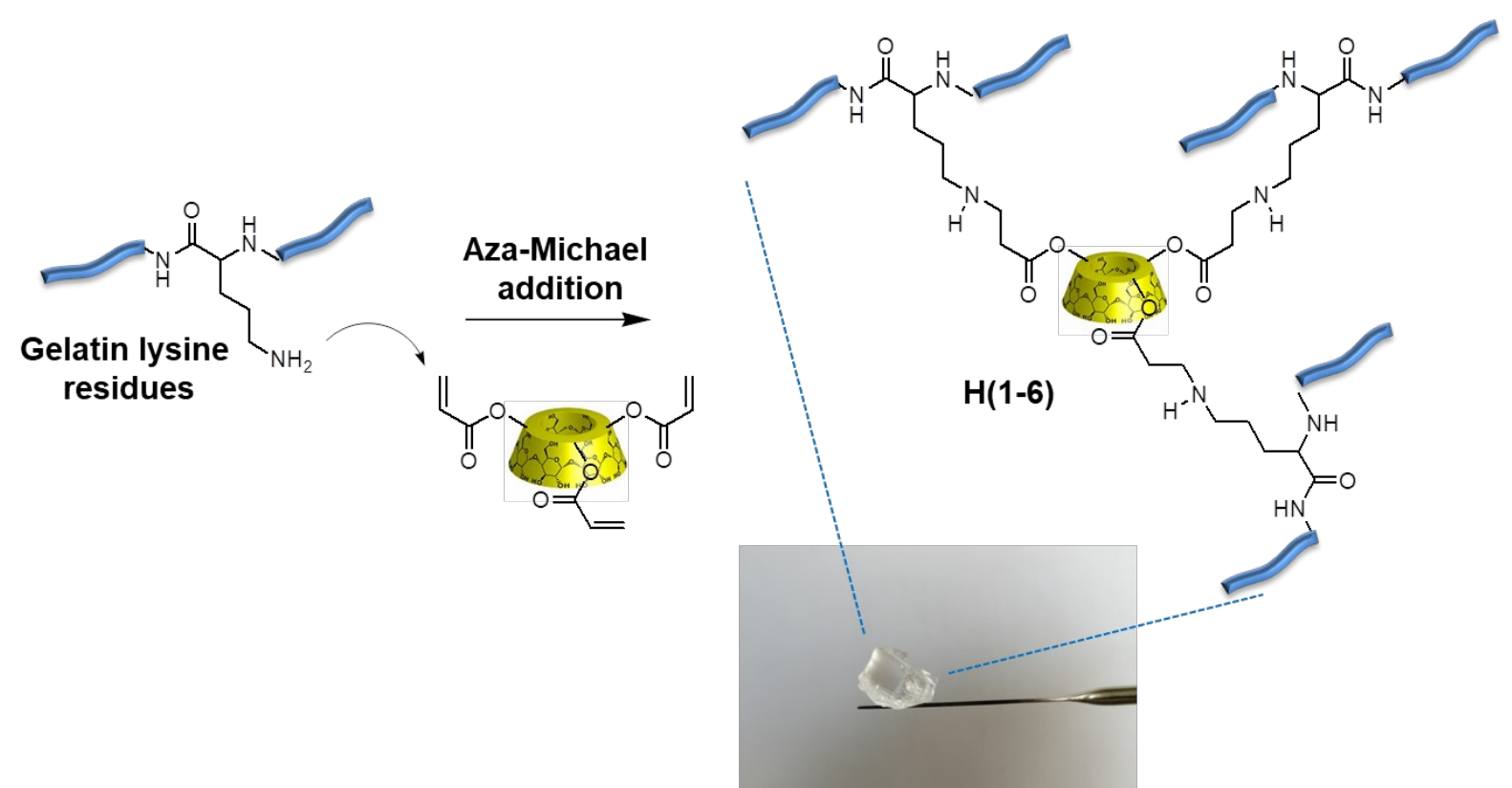

Figure 2. Crosslinking mechanism via multi-addition of gelatin amino groups onto acrylate functionalities of $\beta-C D$-acrylate. Representative picture of transparent hydrogel $\mathrm{H}-4$.

A series of hydrogels composed of different $\beta-C D$ acrylate crosslinker ratio were synthesized in aqueous PBS buffer environment at $37{ }^{\circ} \mathrm{C}$ reaction temperature. Due to the multiple additions of amine groups onto acryloyl functionalities, relatively fast network formation was established within minutes. To ensure maximum available crosslinking, gelation reactions were continued for $6 \mathrm{~h}$. Although the efficiency of the crosslinking process was prominent in uncatalyzed conditions (Table 1, Entry 1 and 2), gel formation was further promoted by using organo-catalysts DBU (Table 1, Entry 3-6). In aqueous conditions, $\beta-C D$ is an efficient catalyst for aza-Michael addition of various amines onto electron-deficient carbonyls. (40) On the other hand, DBU possesses superior promotion of nitrogen nucleophile reactivity towards a, $\beta$-unsaturated carbonyl compounds. (41)

A strong hydrogenation reaction was confirmed by FT-IR characterization of reaction ingredients and resulting hydrogels (Figure 3 ). The spectrum of $\beta$ CD-acrylate shows characteristic vibrations at 1720 $\mathrm{cm}^{-1}$ and $1664 \mathrm{~cm}^{-1}$ due to the stretchings of $\mathrm{C}=\mathrm{O}$ and $\mathrm{C}=\mathrm{C}$, respectively. In the FT-IR spectrum of gelatin, typical amide peaks are resolved at 1639 $\mathrm{cm}^{-1}$ and $1535 \mathrm{~cm}^{-1}$. The spectrum of hydrogel $\mathrm{H}-4$ is characterized by the presence of the typical vibration peaks of both gelatin and $\beta-C D$-acrylate, demonstrating the structural integration of the hydrogelation components into the matrix network. 


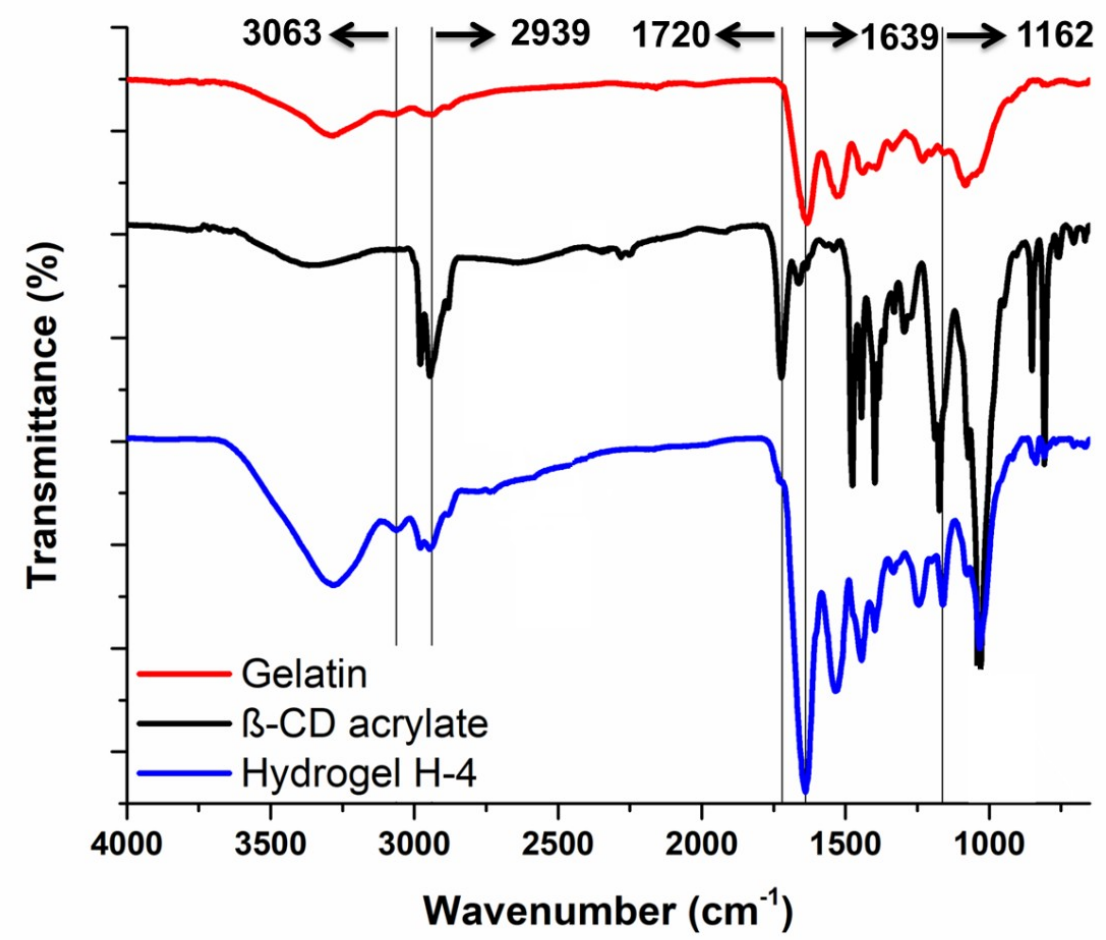

Figure 3. FT-IR structural analysis of gelatin, $\beta$-CD-acrylate, and hydrogel $\mathrm{H}-4$.

The properties of fabricated hydrogels are summarized in Table 1 . As expected, increased $\beta$ CD-acrylate feed in gel formulation resulted in higher gel conversions. The catalytic introduction of DBU in the gelation process has led to enhanced crosslinking efficiencies in different $\beta-C D$-acrylate feeds. Obtained hydrogels are clear transparent samples in the water-swollen state (Figure 2). The morphological characterization of hydrogels using SEM analysis on freeze-dried samples revealed interconnected porous structures with varying pore sizes (Figure 4).

Table 1. Properties of hydrogels with varying gelatin / $\beta-C D-A c$ ratio.

\begin{tabular}{|c|c|c|c|c|c|}
\hline Entry & Hydrogel $^{a}$ & $\begin{array}{c}\text { Feed } \\
\text { Gelatin (mg) : } \beta-C D- \\
\text { acrylate (mg) }\end{array}$ & $\begin{array}{c}\text { Gel Conv. } \\
(\%)\end{array}$ & $\begin{array}{c}\text { ESR } \\
(\times 100 \%)\end{array}$ & $\begin{array}{c}\text { Drug Load } \\
\text { (mg/g dry gel) }\end{array}$ \\
\hline 1 & $\mathrm{H}-1$ & $100.0: 5.0$ & 67 & - & - \\
\hline 2 & $\mathrm{H}-2$ & $100.0: 10.0$ & 71 & - & - \\
\hline 3 & $\mathrm{H}-3$ & $100.0: 5.0$ & 73 & $9.4( \pm 2.7)$ & $11.9( \pm 4.4)$ \\
\hline 4 & $\mathrm{H}-4$ & $100.0: 10.0$ & 77 & $6.7( \pm 2.1)$ & $18.7( \pm 5.8)$ \\
\hline 5 & $\mathrm{H}-5$ & $100.0: 15.0$ & 82 & $5.8( \pm 1.8)$ & $27.1( \pm 5.3)$ \\
\hline 6 & $\mathrm{H}-6$ & $100.0: 20.0$ & 87 & $3.9( \pm 1.3)$ & $31.7( \pm 7.4)$ \\
\hline
\end{tabular}

${ }^{\mathrm{a}} \mathrm{H}-1$ and $\mathrm{H}-2$ : No organo-catalyst; $\mathrm{H}(3-6)$ : DBU catalyst. 

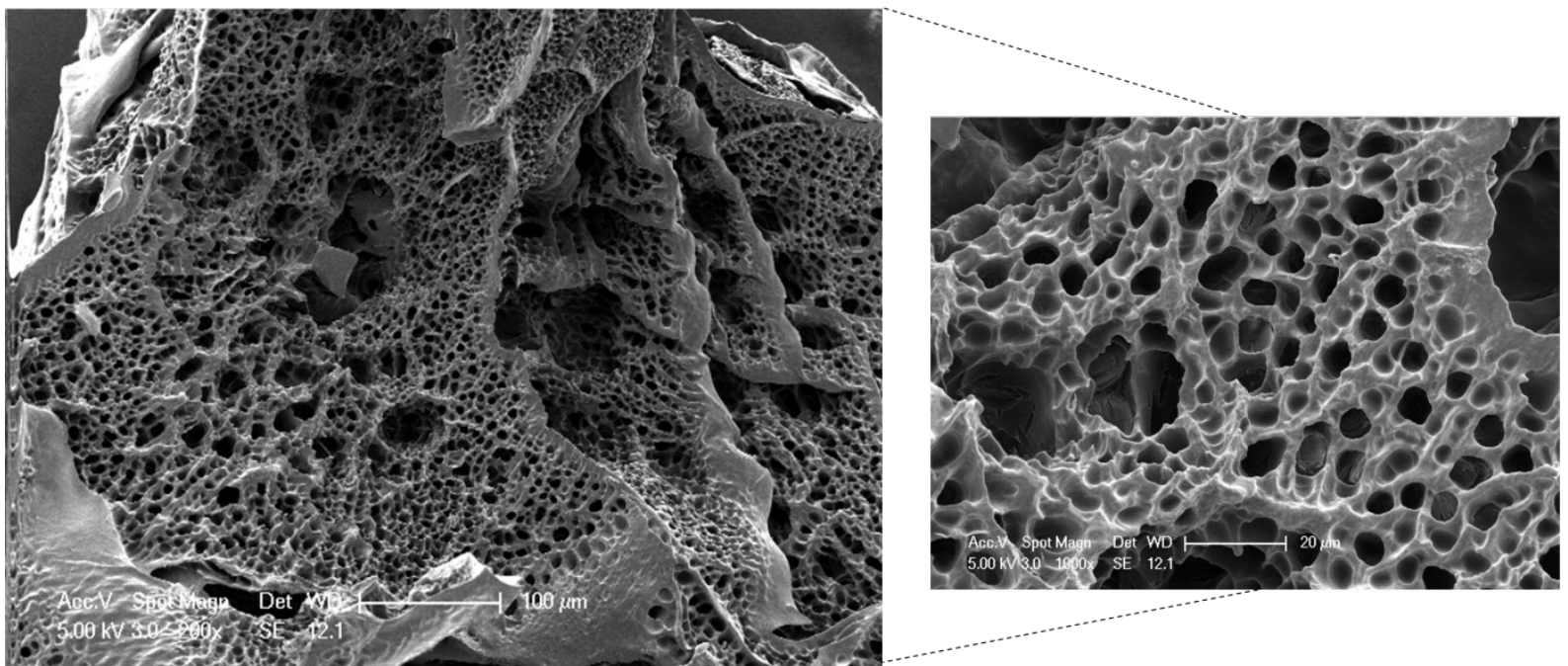

Figure 4. Representative SEM images of freeze-dried hydrogel $\mathrm{H}-4$ with two different magnification (Scale bars: $100 \mu \mathrm{m}$ for larger image and $20 \mu \mathrm{m}$ for smaller image).

Equilibrium water uptake ratio of a hydrogel is an essential physical property in addressing gel mechanical properties, surface and network properties, and diffusion of solutes. (1) Though various parameters (i.e., $\mathrm{pH}$, temperature) can contribute to ascertain the water uptake, maximum swelling is mostly related to the nature of polymer matrix and crosslinking degree. The swelling properties of the fabricated hydrogels were investigated in aqueous PBS conditions $(\mathrm{pH}: 7.4,25$ ${ }^{\circ} \mathrm{C}$ ). All the hydrogels displayed pronounced swelling degrees ( 940-390 \% to the mass of gel sample) because of the hydrophilic groups present on the gelatin backbone and cyclodextrin crosslinker (Figure 5). Equilibrium water uptakes were reached in relatively short periods in applied experimental conditions. It was observed that hydrogels exhibit maximum water uptake dependency on the feed ratio of the gelatin matrix and $\beta-C D$-acrylate crosslinker. Relatively higher maximum swelling degrees were observed in case of a lower crosslinker ratio in the feed. This can be attributed to higher free gel volumes and voids of network possessed by lower crosslinking degree.

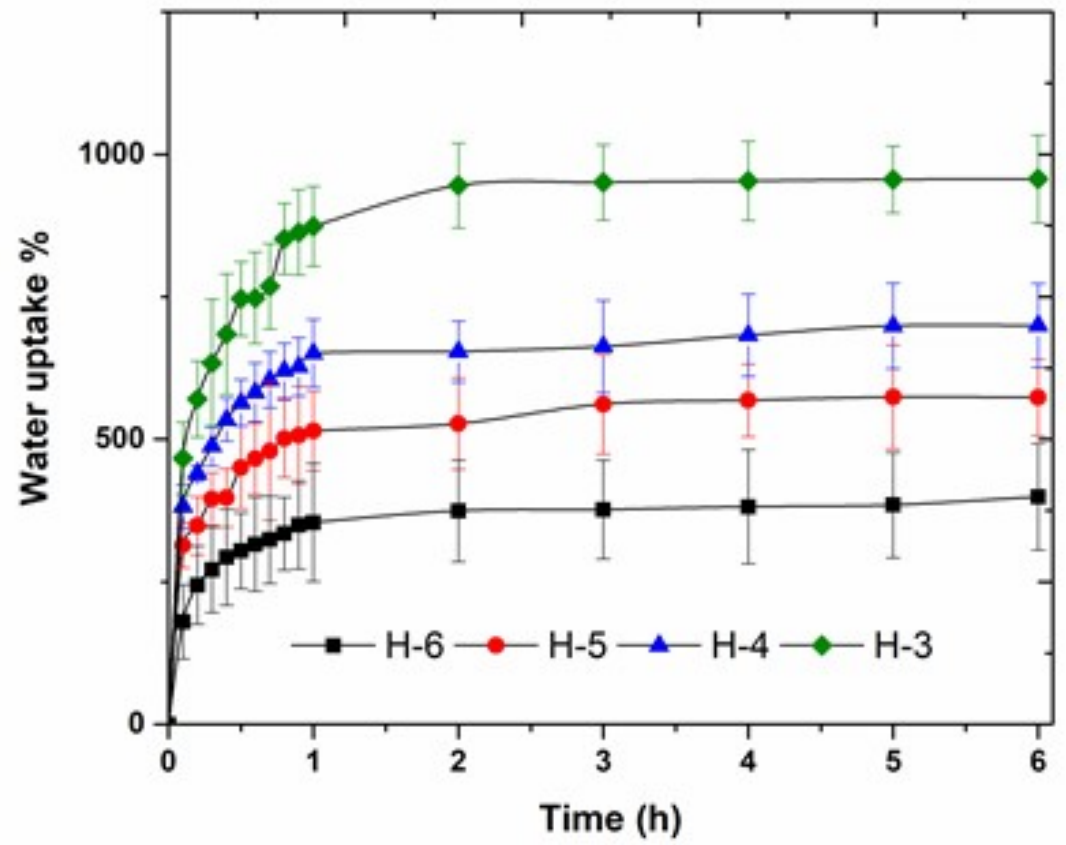

Figure 5. Equilibrium swelling profiles of gelatin-based $\beta-C D$ containing hydrogels in PBS buffer.

Hydrogel viscoelastic properties were examined via dynamic frequency scan analysis of prepared hydrogels at $37^{\circ} \mathrm{C}$. The tests of hydrogels having different crosslinker ratios were performed at a constant $1.0 \%$ strain between $0.05-100 \mathrm{rad} / \mathrm{s}$. As it can be seen in Figure 6, all samples exhibited permanent elastic character in the test frequency interval where storage moduli $\left(G^{\prime}\right)$ were higher than 
loss moduli ( $G^{\prime \prime}$ ). According to the results, $G^{\prime}$ and $G$ " values of the samples show relatively low oscillation frequency dependency maintaining highly homogenous crosslinking of gel networks. (42) An increase in the ratio of $\beta C D$-based crosslinker has resulted in higher moduli, which could be due to the increased network structure. The damping factors ( $\tan \delta, G^{\prime \prime} / G^{\prime}$ ) of hydrogels were less than 1 in all cases representing the higher elastic character of networks over viscous behavior. (43) a)

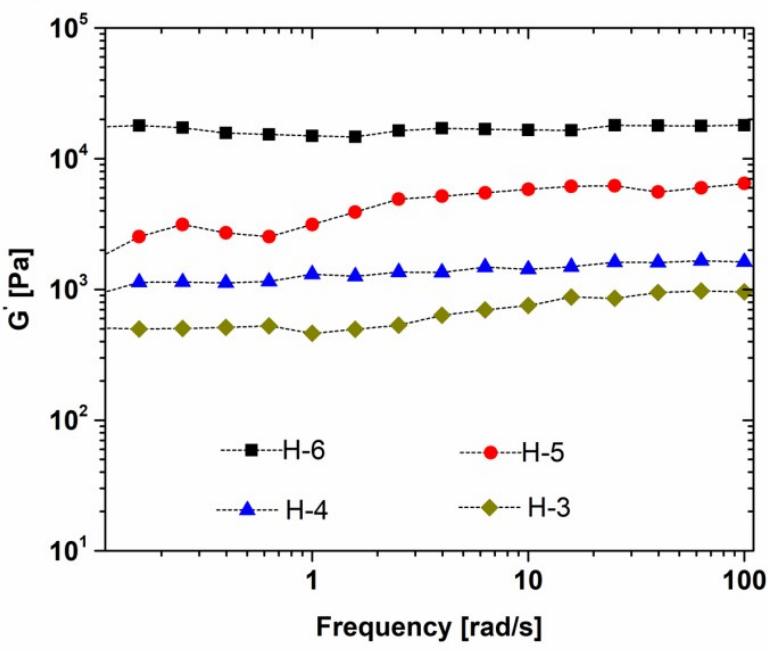

b)

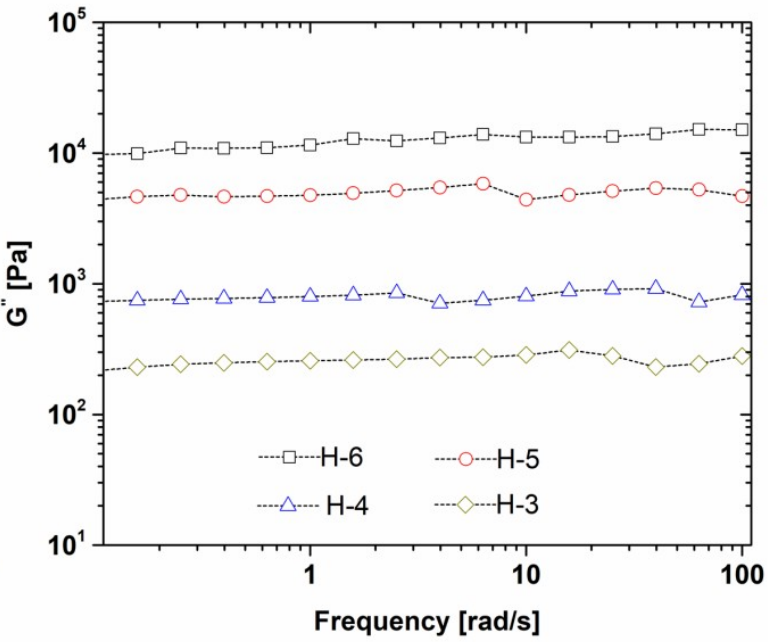

Figure 6. Dynamic frequency scan analysis of hydrogels: a) Storage and b) loss moduli.

\section{Drug loading and release studies}

Loading and sustained/controlled release of drugs, biomolecules, and other therapeutically relevant materials using hydrogels procure great potentials in realizing effective administration systems. The typical strategy for loading and release of drugs is based on the diffusion phenomena governed by weak interactions of the hydrogel matrix with subject molecules. In hydrogel networks that accommodate cyclodextrins as molecular buckets, inclusion complexation between hydrophobic molecules and cyclodextrins ensures another means of drug loading. Diclofenac-Na, widely used as a non-steroidal anti-inflammatory drug for various complaints and diseases, was employed as a model hydrophobic molecule to study the drug loading and release studies of fabricated hydrogels. It has been established that diclofenac- $\mathrm{Na}$ can form a molecular inclusion complex with $\beta$-cyclodextrin. (44) Hydrogels prepared as disk-shaped samples were loaded with the drug by solution absorption at 37 ${ }^{\circ} \mathrm{C}$. 0.5 wt. \% soaking solution was used for $24 \mathrm{~h}$ incubation, which is the required time to reach equilibrium as determined by UVspectrophotometry. The drug loading efficiencies of hydrogels determined from the difference between initial and final soaking concentrations were given in Table 1. According to the results, the loaded drug amount increased from $11.9(\mathrm{mg} / \mathrm{g})$ to $31.7(\mathrm{mg} / \mathrm{g})$ for different hydrogels. A direct relation between crosslinker amount and drug levels was accounted for, where increased $\beta$-CD-acrylate crosslinker in the hydrogel resulted in considerably higher drug loading. The enhanced drug loading could be due to the higher available binding sites of cyclodextrin cavities to complex with diclofenac-Na molecules. The inclusion complexation might have increased the solubility of slightly hydrophobic drug molecules within the hydrophilic gel network.

In vitro drug release profiles of the hydrogels containing different $\beta$-CD-acrylate crosslinker ratios were investigated in PBS buffer $(\mathrm{pH}: 7.4)$ at $37^{\circ} \mathrm{C}$. Initially, drug-loaded hydrogels taken from soaking solutions were gently washed with PBS buffer and then placed in the release medium. Aliquots of the release medium were taken out and replaced with the fresh solution at pre-determined intervals to resemble the physiological conditions. Timedependent release profiles determined via UVspectrophotometry were given in Figure 7. Overall, hydrogel $\mathrm{H}-3$ having the lowest $\beta-\mathrm{CD}$-acrylate, released its content faster than other hydrogels. An initial accelerated release was observed for all four hydrogels, which could be attributed to the burst removal of drug molecules present in the aqueous phase and adsorbed on the network matrix. (45) As expected, H-6 with the highest crosslinker content showed the lowest initial burst release and followed by $\mathrm{H}-4$ and $\mathrm{H}-5$. Incorporation of higher $\beta-\mathrm{CD}-$ acrylate caused a higher crosslinking degree of the network as well as enhanced drug/cyclodextrin complexation interactions, yielding slow diffusion of diclofenac-Na from the hydrogels. After the initial burst releases, all gelatin-based hydrogels exhibited sustained release profiles over a couple of days. In a specific time, relatively slower and lower total drug release from higher cyclodextrin content hydrogel could be ascribed to enhanced molecular inclusion interactions. 
In an overall structure-property relationship of fabricated hydrogels, it was observed that the amount of $\beta-C D$ content in the hydrogels maintained enhanced drug/matrix interactions. Hydrogel physical properties can also be manipulated by adjusting the crosslinking degrees, which of them are simply controlled by the stoichiometric ratios of gelatin and $\beta-C D$-acrylate in gel formulations. The efficient covalent crosslinking reaction allows in situ gel formation that might improve the versatility of material to injectable drug release systems. One can suggest that the demonstrated approach could be utilized in the design and synthesis of hydrogelbased controlled/sustained drug delivery systems.

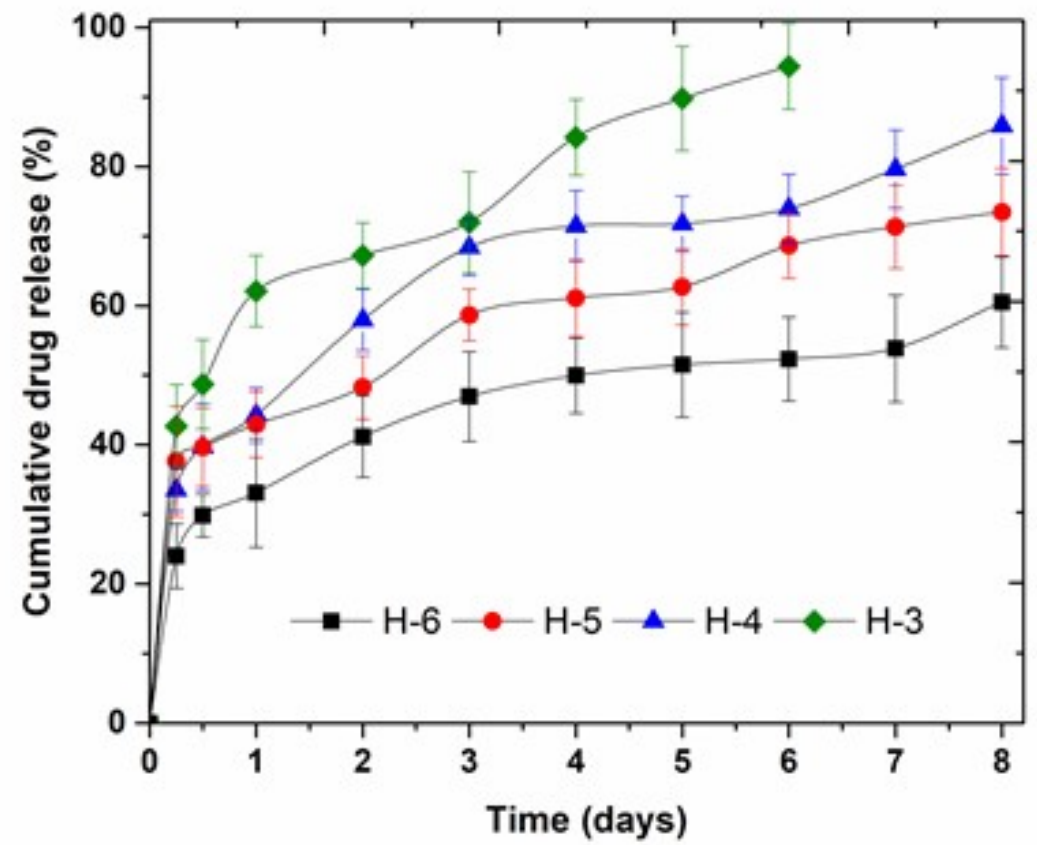

Figure 7. Cumulative release profiles of diclofenac-Na from gelatin-based $\beta-C D$ containing hydrogels at 37 ${ }^{\circ} \mathrm{C}$ in PBS buffer $(\mathrm{pH} 7.4)$. Data with mean $\pm S D(n=3)$.

\section{CONCLUSION}

A series of gelatin-based hydrogels incorporating $\beta$ $C D$ units were fabricated under benign reaction conditions. Chemical crosslinking was established by utilizing unmodified pristine porcine gelatin via azaMichael addition reaction. Practical and straightforward synthesis methodology allowed tunable control of the $\beta$-CD-based crosslinker amount in gel networks. It was shown that hydrogel physical properties could be tuned by adjusting the stoichiometry between amine and acrylate groups of precursor materials. The potential sustained drug release application of hydrogels was investigated by employing diclofenac-Na as a model hydrophobic molecule capable of inclusion complex formation with $\beta-C D$. The results suggested that the amount of $\beta-C D$ content in the hydrogels maintained enhanced drug/matrix interactions leading to higher drug loading into the gel network. Lower initial burst releases and more sustained drug release for days was also attained in case of higher $\beta-C D$ incorporation. It is believed that the efficient covalent crosslinking reaction demonstrated here could be utilized in the fabrication of biologically relevant gelatin and cyclodextrin based biomaterials.

\section{REFERENCES}

1. Peppas NA, Hilt JZ, Khademhosseini A, Langer R Hydrogels in biology and medicine: From molecular principles to bionanotechnology. Advanced Materials. 2006. doi:10.1002/adma.200501612

2. Caló $E$, Khutoryanskiy V V. Biomedical applications of hydrogels: A review of patents and commercial products. European Polymer Journal. 2015. doi:10.1016/j.eurpolymj.2014.11.024

3. Hoffman AS. Hydrogels for biomedical applications. Advanced Drug Delivery Reviews. 2012.

doi:10.1016/j.addr.2012.09.010

4. Lee KY, Mooney DJ. Hydrogels for tissue engineering. Chemical Reviews. 2001. doi:10.1021/cr000108x

5. Wang $\mathrm{H}$, Heilshorn SC. Adaptable Hydrogel Networks with Reversible Linkages for Tissue Engineering. Advanced Materials. 2015. doi:10.1002/adma.201501558

6. Geckil H, Xu F, Zhang X, Moon S, Demirci U. Engineering hydrogels as extracellular matrix mimics. Nanomedicine. 2010. doi:10.2217/nnm.10.12

7. Li J, Mooney DJ. Designing hydrogels for controlled drug delivery. Nature Reviews Materials. 2016. doi:10.1038/natrevmats.2016.71 
8. Qiu Y, Park K. Environment-sensitive hydrogels for drug delivery. Advanced Drug Delivery Reviews. 2012. doi: 10.1016/j.addr.2012.09.024

9. Hoare TR, Kohane DS. Hydrogels in drug delivery: Progress and challenges. Polymer. 2008.

doi: $10.1016 /$ j.polymer.2008.01.027

10. Yang JA, Yeom J, Hwang BW, Hoffman AS, Hahn SK. In situ-forming injectable hydrogels for regenerative medicine. Progress in Polymer Science. 2014. doi:10.1016/j.progpolymsci.2014.07.006

11. Yu L, Ding J. Injectable hydrogels as unique biomedical materials. Chemical Society Reviews. 2008. doi: 10.1039/b713009k

12. Li Y, Rodrigues J, Tomás H. Injectable and biodegradable hydrogels: Gelation, biodegradation and biomedical applications. Chemical Society Reviews. 2012. doi: $10.1039 /$ c1cs15203c

13. Tiller JC. Increasing the local concentration of drugs by hydrogel formation. Angewandte Chemie -

International Edition. 2003. doi:10.1002/anie.200301647

14. Cai S, Liu Y, Xiao ZS, Prestwich GD. Injectable glycosaminoglycan hydrogels for controlled release of human basic fibroblast growth factor. Biomaterials. 2005. doi: 10.1016/j.biomaterials.2005.03.012

15. Paul A, Hasan A, Kindi H Al, Gaharwar AK, Rao VTS Nikkhah $M$, et al. Injectable graphene oxide/hydrogelbased angiogenic gene delivery system for vasculogenesis and cardiac repair. ACS Nano. 2014 doi: $10.1021 / \mathrm{nn} 5020787$

16. Yue K, Santiago GT, Tamayol A, Annabi N, Khademhosseini A, Hospital W, et al. Synthesis, properties, and biomedical applications of gelatin methacryloyl (GelMA) hydrogels. Biomaterials. 2016. doi:10.1016/j.biomaterials.2015.08.045.Synthesis

17. Olsen D, Yang C, Bodo M, Chang R, Leigh S, Baez J, et al. Recombinant collagen and gelatin for drug delivery. Advanced Drug Delivery Reviews. 2003. doi: $10.1016 /$ j.addr.2003.08.008

18. Yue K, Trujillo-de Santiago G, Alvarez MM, Tamayol A, Annabi N, Khademhosseini A. Synthesis, properties, and biomedical applications of gelatin methacryloyl (GelMA) hydrogels. Biomaterials. 2015. doi: $10.1016 /$ j.biomaterials. 2015.08 .045

19. Davidenko N, Schuster CF, Bax D V., Farndale RW, Hamaia S, Best SM, et al. Evaluation of cell binding to collagen and gelatin: a study of the effect of 2D and 3D architecture and surface chemistry. J Mater Sci Mater Med. 2016. doi:10.1007/s10856-016-5763-9

20. Gasperini L, Mano JF, Reis RL. Natural polymers for the microencapsulation of cells. Journal of the Royal Society Interface. 2014. doi:10.1098/rsif.2014.0817

21. Chen YC, Lin RZ, Qi H, Yang $Y$, Bae $H$, Melero-Martin $\mathrm{JM}$, et al. Functional human vascular network generated in photocrosslinkable gelatin methacrylate hydrogels.

Advanced Functional Materials. 2012. doi: $10.1002 /$ adfm. 201101662
22. Pepelanova I, Kruppa K, Scheper T, Lavrentieva A. Gelatin-methacryloyl (GelMA) hydrogels with defined degree of functionalization as a versatile toolkit for 3D cell culture and extrusion bioprinting. Bioengineering. 2018. doi: $10.3390 /$ bioengineering 5030055

23. Young S, Wong M, Tabata $Y$, Mikos AG. Gelatin as a delivery vehicle for the controlled release of bioactive molecules. Journal of Controlled Release. 2005. doi:10.1016/j.jconrel.2005.09.023

24. Draye JP, Delaey B, Van De Voorde A, Van Den Bulcke $A$, De Reu B, Schacht E. In vitro and in vivo biocompatibility of dextran dialdehyde cross-linked gelatin hydrogel films. Biomaterials. 1998. doi:10.1016/S01429612(98)00049-0

25. Koshy ST, Desai RM, Joly P, Li J, Bagrodia RK, Lewin $\mathrm{SA}$, et al. Click-Crosslinked Injectable Gelatin Hydrogels. Advanced Healthcare Materials. 2016. doi: 10.1002/adhm.201500757

26. Chen B, Hu X. An injectable composite gelatin hydrogel with $\mathrm{pH}$ response properties. Journal of Nanomaterials. 2017. doi: $10.1155 / 2017 / 5139609$

27. Arslan M, Sanyal R, Sanyal A. Cyclodextrin embedded covalently crosslinked networks: Synthesis and applications of hydrogels with nano-containers. Polymer Chemistry. 2020. doi:10.1039/c9py01679a

28. Arslan M, Sanyal R, Sanyal A. Cyclodextrin-containing hydrogel networks. In: Mishra M, editor. Encyclopedia of Biomedical Polymers and Polymeric Biomaterials. Taylor and Francis: New York; 2015. p. 2243-58. Available from: https://www.taylorfrancis.com/books/e/9781466501799/c hapters/10.1081\%2FE-EBPP-120050543

29. Arslan M, Aydin D, Degirmenci A, Sanyal A, Sanyal R. Embedding well-defined responsive hydrogels with nanocontainers: Tunable materials from telechelic polymers and cyclodextrins. ACS Omega. 2017. doi:10.1021/acsomega.7b00787

30. Arslan M, Gevrek TN, Sanyal A, Sanyal R. Cyclodextrin mediated polymer coupling via thiol-maleimide conjugation: Facile access to functionalizable hydrogels. RSC Advances. 2014. doi:10.1039/c4ra12408a

31. Arslan M, Gevrek TN, Sanyal R, Sanyal A. Fabrication of poly(ethylene glycol)-based cyclodextrin containing hydrogels via thiol-ene click reaction. European Polymer Journal. 2015. doi:10.1016/j.eurpolymj.2014.08.018

32. Arslan M, Yirmibesoglu T, Celebi M. In situ Crosslinkable Thiol-ene Hydrogels Based on PEGylated Chitosan and $\beta$-Cyclodextrin, J. Turk. Chem. Soc., Sect. A: Chem., 2018, 5, 1327-1336.

33. Slavkova M Iv, Momekova DB, Kostova BD, Momekov G Tz, Petrov PD, Bulgarian Chemical Communications, 2017, Volume 49, Number 4, 792 - 799.

34. Hafidz RNRM, Yaakob CM, Amin I, Noorfaizan A. Chemical and functional properties of bovine and porcine skin gelatin. Int Food Res J. 2011.

35. Bertoldo M, Bronco S, Gragnoli T, Ciardelli F. Modification of gelatin by reaction with $1,6-$ diisocyanatohexane. Macromol Biosci. 2007. doi:10.1002/ 
mabi. 200600215

36. De Carvalho RA, Grosso CRF. Characterization of gelatin based films modified with transglutaminase, glyoxal and formaldehyde. Food Hydrocoll. 2004.

doi:10.1016/j.foodhyd.2003.10.005

37. Honda I, Arai K, Mitomo H. Characterization of crosslinks introduced in gelatin. J Appl Polym Sci. 1997.

doi:10.1002/(SICI)1097-

4628(19970606)64:10<1879: :AID-APP2>3.0.CO;2-F

38. Bigi A, Cojazzi G, Panzavolta S, Roveri N, Rubini K. Stabilization of gelatin films by crosslinking with genipin. Biomaterials. 2002. doi:10.1016/S0142-9612(02)00235-1

39. Liu C, Zhang Z, Liu X, Ni X, Li J. Gelatin-based hydrogels with $\beta$-cyclodextrin as a dual functional component for enhanced drug loading and controlled release. RSC Advances. 2013. doi:10.1039/c3ra42532k

40. Surendra K, Krishnaveni NS, Sridhar R, Rao KR. $\beta$ Cyclodextrin promoted aza-Michael addition of amines to conjugated alkenes in water. Tetrahedron Letters. 2006. doi: $10.1016 /$ j.tetlet.2006.01.124
41. Yeom CE, Kim MJ, Kim BM. 1,8-

Diazabicyclo[5.4.0]undec-7-ene (DBU)-promoted efficient and versatile aza-Michael addition. Tetrahedron. 2007. doi:10.1016/j.tet.2006.11.037

42. Siemoneit $U$, Schmitt C, Alvarez-Lorenzo C, Luzardo A, Otero-Espinar F, Concheiro A, et al. Acrylic/cyclodextrin hydrogels with enhanced drug loading and sustained release capability. Int J Pharm. 2006. doi:10.1016/j.ijpharm.2005.12.046

43. Jin R, Moreira Teixeira LS, Dijkstra PJ, Karperien M, van Blitterswijk CA, Zhong $Z Y$, et al. Injectable chitosanbased hydrogels for cartilage tissue engineering.

Biomaterials. 2009.

doi:10.1016/j.biomaterials.2009.01.020

44. Das S, Subuddhi U. Studies on the complexation of diclofenac sodium with $\beta$-cyclodextrin: Influence of method of preparation. J Mol Struct. 2015. doi:10.1016/j.molstruc.2015.07.001

45. Huang X, Brazel CS. On the importance and mechanisms of burst release in matrix-controlled drug delivery systems. Journal of Controlled Release. 2001. doi:10.1016/S0168-3659(01)00248-6 


\title{
SUPPORTING INFORMATION
}

\section{In situ Crosslinking System of Gelatin with Acrylated $\beta$-cyclodextrin Towards the Fabrication of Hydrogels for Sustained Drug Release}

\author{
Mehmet Arslan \\ University of Yalova, Faculty of Engineering, Department of Polymer Materials Engineering, 77200, Yalova, \\ Turkey.
}

Synthesis of Acryloyl-modified $\boldsymbol{\beta}$-cyclodextrin ( $\boldsymbol{\beta}$-CD-acrylate): Synthesis of the $\beta$-CD-acrylate was conducted by following a previously reported procedure.(1) $\beta-C D(1.0 \mathrm{~g}, 0.88 \mathrm{mmol})$ previously dried by lyophilization, and triethylamine $(0.5 \mathrm{~mL}, 6.16 \mathrm{mmol})$ were dissolved in $10 \mathrm{~mL}$ dry dimethylformamide. The mixture was cooled to $0{ }^{\circ} \mathrm{C}$ in an ice bath and acryloyl chloride $(0.85 \mathrm{~mL}, 6.16 \mathrm{mmol})$ was added dropwise. The reaction was continued for $16 \mathrm{~h}$ at room temperature. After the reaction was complete, a white precipitate was removed by filtration. The purified product was obtained by precipitation of the mixture into cold acetone and filtration (Yield: 41\%). The structure of the compound is consistent with the reported literature data (Figure S1). Degree of acetylation based on peak integrals of $\mathrm{H} 1$ protons at $5.03 \mathrm{ppm}$ and vinyl protons at $5.82-6.40 \mathrm{ppm}$ revealed average degree of acetylation $\sim 5.9$ per molecule.

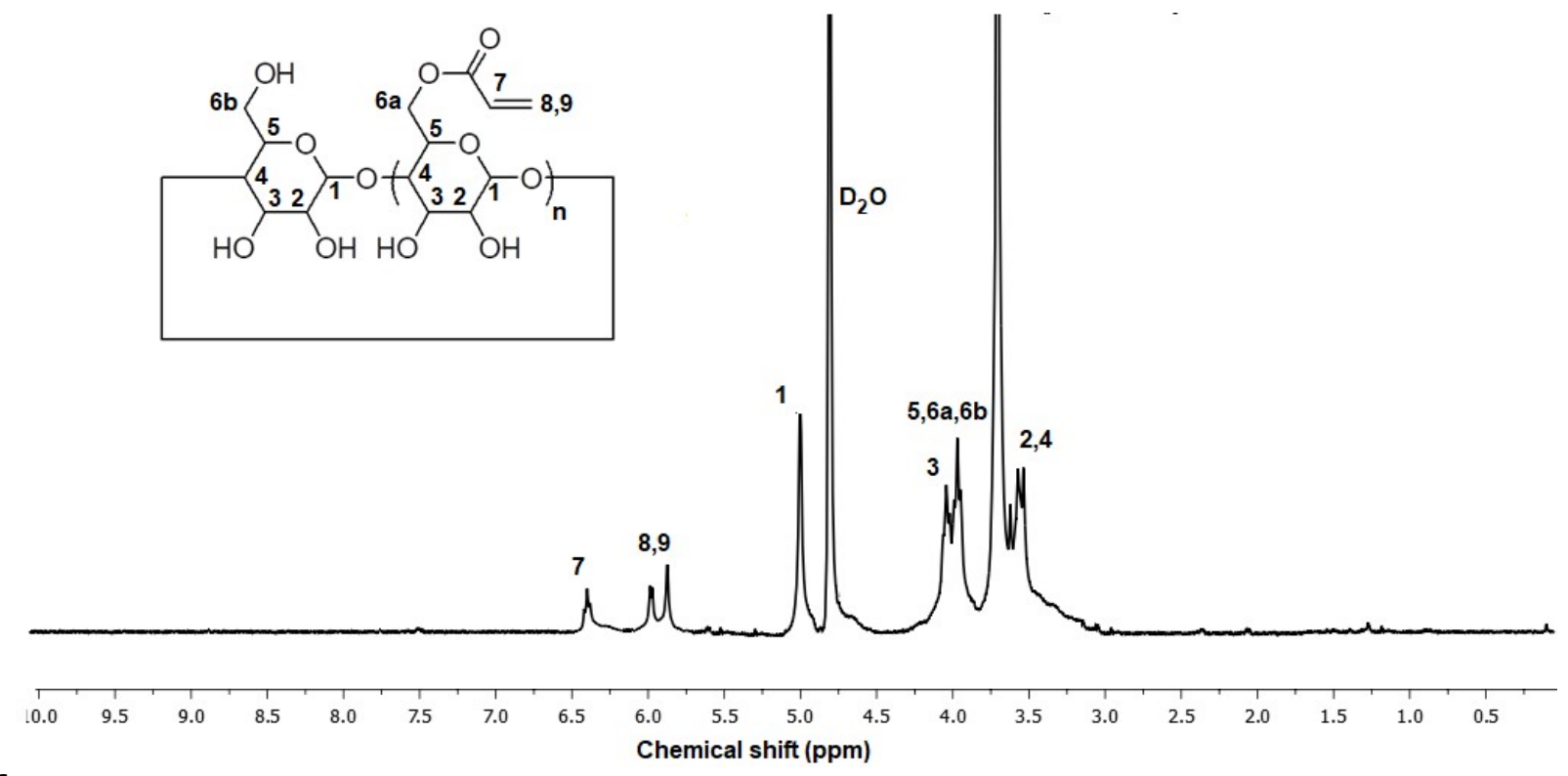

References:

1. Slavkova M IV, Momekova DB, Kostova BD, Momekov G Tz, Petrov PD, Bulgarian Chemical Communications, 2017, Volume 49, Number 4, 792 - 799. 